\title{
Correlation of Tumor Location to Clinical Outcomes in Colorectal Cancer: A Single-institution Retrospective Analysis
}

\author{
CARLO SIGNORELLI $^{1}$, MARIO GIOVANNI CHILELLI ${ }^{1}$, ISABELLA SPERDUTI ${ }^{2}$, SILVANA GIACINTI $^{1}$, \\ PIETRO MARIA AMODIO ${ }^{3}$, RAFFAELE MACARONE PALMIERI ${ }^{3}$, TERESA VALENTINA RANALLI ${ }^{4}$, \\ VITO VINCENZO GOMES ${ }^{4}$, MARIA ELENA ROSETTO ${ }^{5}$, FABRIZIO NELLI ${ }^{1}$ and ENZO MARIA RUGGERI ${ }^{1}$ \\ ${ }^{1}$ Medical Oncology Unit, Belcolle Hospital, ASL Viterbo, Viterbo, Italy; \\ ${ }^{2}$ Department of Bio-Statistics, Regina Elena National Cancer Institute, Rome, Italy; \\ ${ }^{3}$ General Oncological Surgery, Belcolle Hospital, ASL Viterbo, Viterbo, Italy; \\ ${ }^{4}$ Pathology Unit, Belcolle Hospital, ASL Viterbo, Viterbo, Italy; \\ ${ }^{5}$ Radiotherapy Unit, Belcolle Hospital, ASL Viterbo, Viterbo, Italy
}

\begin{abstract}
Background/Aim: Recent data highlighted that location of metastatic colorectal cancer ( $m C R C$ ) may have a prognostic impact and also a predictive value of the outcomes of first-line therapy. Materials and Methods: The records of $m C R C$ patients who underwent first-line therapy from 2011 to April 2018 at our Institute were retrospectively reviewed. Progression-free survival (PFS), overall survival $(O S)$ and objective response rate (ORR) according to the primary tumor location were investigated. Results: Overall, 130 patients were eligible. Two-year OS was $82.9 \%$ in leftsided colon cancers (LCC) and $67.5 \%$ in right-sided (RCC) $(p=0.32)$. One-year mPFS was statistically longer in LCC (46.8\% vs. $24.2 \%, p=0.0005)$. mPFS was longer in LCC treated with anti-VEGF vs. anti-EGFR $(p=0.06)$. ORR was $51.1 \%$ in LCC, $25 \%$ in RCC ( $p=0.008)$. Overall, 11 complete responses all in LCC were observed $(p=0.03)$. Conclusion: Tumor location has a prognostic impact and might influence the outcomes of $m C R C$ patients.
\end{abstract}

In the era of personalized management of metastatic colorectal cancer, there is a general consensus that anatomical location may have a prognostic impact and also a predictive value on the outcomes of first-line therapy with monoclonal antibodies (mAbs) and cytotoxic agents in patients (pts) with metastatic colorectal cancer (mCRC). A significant amount of data from advances in the treatment of mCRC help us better understand the biology of this disease

Correspondence to: Dr Carlo Signorelli, Medical Oncology Unit, Belcolle Hospital, ASL Viterbo, 01100 Viterbo, Italy. E-mail: carlo.signorelli@asl.vt.it

Key Words: Tumor location, metastatic colorectal cancer, first-line therapy. and individualize regimen planning. $\mathrm{mCRC}$ is a heterogeneous disease that appears to be responsible for the third highest incidence rate, with about 1,849,518 (10.2\%) new cases in 2018, in both sexes and all ages and for the second highest mortality rate, with about $880,792(9.2 \%)$ deaths in 2018, in both sexes and all ages (1). About 50-60\% of patients diagnosed with colorectal cancer develop metastases, most commonly at the liver. LCC patients tend to have hepatic and pulmonary metastases while RCC patients have peritoneal secondary metastases that determine the worst outcome $(2,3)$. Several studies have consistently reported that left-side is associated with better prognosis with an overall survival strictly depended on the stage of cancer and the presence of metastases $(4,5)$. The clinical management of this disease with first-line doublets plus tailored biological agents that takes into account mutational characterization, has further improved survival to more than 32 months $(6,7)$. The pathogenesis of colorectal cancer depends on the anatomical position of the tumor and differs between the right and left sides of the colon. Tumors arising from different sides of the colon are clinically and molecularly distinct, exhibit different features, such as epidemiological incidence, physiological characteristics, molecular alterations, and even survival outcome and are more and more considered as separate tumor types $(8,9)$. Bufill has first proposed in 1990, that right and left colon tumors may arise from different biological pathways and represent distinct genetic entities (10). In particular, carcinomas of the left colon (those originating from splenic flexure, descending colon, sigmoid colon, rectum or onethird of the transverse colon) derive from the embryonic hindgut; on the contrary, carcinomas of the right colon (those originating from the appendix, the blind, the ascending colon, hepatic flexion or two-thirds of the transverse colon) derive from the embryonic midgut. Clinically, this 
heterogeneity is very useful in therapeutic decision-making and in helping to predict and prognosticate the results. The molecular factors that predict the effectiveness of CRC treatment are largely unknown; the mutational state of $R A S$ and perhaps $B R A F$ are currently the only references in the decision-making process of systemic therapy. The principal differences between right- and left-sided primary colon cancers are listed in Figure 1 (11-15). The objective of this current investigation was principally to evaluate the prognostic and predictive effect of primary tumor location on clinical outcomes of first-line therapy in previously untreated patients with mCRC, using retrospectively collected data from 2011 to April 2018 at our institute.

\section{Patients and Methods}

For this analysis, 153 patients diagnosed with stage IV colorectal cancer who received first-line chemotherapy with palliative intent were identified and collected from an electronic database pertaining to patient and disease characteristics, treatment and outcomes from January 2011 to April 2018 at our Institute. The enrolled patients met the following inclusion criteria: 1) histologically confirmed adenocarcinoma; 2 ) stage IV colorectal cancer at diagnosis or in the postoperative follow-up; 3) previous administration of adjuvant or neoadjuvant therapies for colorectal cancer stages II-III; 4) first-line therapy with monoclonal antibodies (mAbs) and cytotoxic agents; 5) synchronous or metachronous metastases; 6) known $R A S$ mutational status. Patients with multiple primary tumors, those with primary tumors in both left- and right-site and those treated with curative intent were excluded from the analysis. For the overall study population, primary tumors originating into the appendix, cecum, ascending colon, hepatic flexure and transverse colon were classified as right-sided. Primary tumors originating in the splenic flexure, descending colon, sigmoid colon and rectum were classified as left-sided. The endpoints investigated were OS, PFS, ORR and DFS post-adjuvant treatment. OS (overall survival) was defined as the time from first-line treatment initiation until death from any cause. PFS (progression-free survival) was defined as the time from commencement of first-line therapy until the date of objective disease progression or death from any cause. ORR (objective response rate) including complete and partial responses, was assessed according to RECIST criteria. DFS (disease-free survival or recurrence-free survival) following curative-intent primary therapy, has been defined as the time spent free of signs and symptoms of cancer, from the ending of adjuvant treatment until the first of either recurrence or relapse, second cancer, or death or last follow-up; we divided this interval of time into 3 groups: $<6$ months, 6-12 months, $>12$ months after the completion of adjuvant chemotherapy. Informed consent was waived because of the nature of retrospective study, and confidentiality was maintained for patient data. Patients were followed until 30th of April 2018. The study was approved by the Lazio 1 Ethics Committee (approval no. 937/CE Lazio 1).

Statistical analysis. Descriptive statistics were used to summarize pertinent study information. PFS and OS were calculated by the Kaplan-Meier product limit method from the date of therapy until progression, death due to cancer and/or death from any cause. The
Table I. Baseline patient characteristics and treatment.

\begin{tabular}{|c|c|c|c|c|}
\hline & \multicolumn{4}{|c|}{ Primary tumor location } \\
\hline & \multicolumn{2}{|c|}{$\mathrm{RCC}$} & \multicolumn{2}{|c|}{ LCC } \\
\hline & $\mathrm{N}$ & $\%$ total & $\mathrm{N}$ & $\%$ total \\
\hline Total & 37 & 28.4 & 93 & 71.5 \\
\hline Median age (years) & 66.5 & & 62.3 & \\
\hline \multicolumn{5}{|l|}{ Gender } \\
\hline Female & 16 & 12.3 & 32 & 24.6 \\
\hline Male & 22 & 16.9 & 60 & 46.1 \\
\hline \multicolumn{5}{|l|}{ RAS status } \\
\hline Wild type & 15 & 11.5 & 49 & 37.6 \\
\hline Mutant type & 21 & 16.1 & 40 & 30.7 \\
\hline Not tested & 1 & 0.7 & 4 & 3.07 \\
\hline \multicolumn{5}{|l|}{ Right side } \\
\hline Appendix & 1 & 0.7 & & \\
\hline Cecum & 10 & 7.6 & & \\
\hline Ascending colon & 7 & 5.3 & & \\
\hline Hepatic flexure colon & 3 & 2.3 & & \\
\hline Transverse colon & 8 & 6.1 & & \\
\hline Right colon NOS & 9 & 6.9 & & \\
\hline \multicolumn{5}{|l|}{ Left side } \\
\hline Splenic flexure colon & & & 1 & 0.7 \\
\hline Descending colon & & & 9 & 6.9 \\
\hline Sigmoid colon & & & 22 & 16.9 \\
\hline Rectum-sigmoid junction & & & 16 & 12.3 \\
\hline Rectum & & & 29 & 22.3 \\
\hline Left colon NOS & & & 15 & 11.5 \\
\hline Prior adjuvant therapy & 13 & 10.0 & 45 & 34.6 \\
\hline Metastatic patients at diagnosis & 24 & 18.4 & 48 & 36.9 \\
\hline \multicolumn{5}{|l|}{ Metastatic disease sites } \\
\hline Liver only & 12 & 9.2 & 19 & 14.6 \\
\hline Liver + other & 15 & 11.5 & 30 & 23.0 \\
\hline Other & 11 & 8.4 & 43 & 33.0 \\
\hline \multicolumn{5}{|l|}{ Chemotherapy regimen } \\
\hline Fluoropyrimidine alone & 6 & 4.6 & 6 & 4.6 \\
\hline Doublet chemotherapy & 31 & 23.8 & 82 & 63.0 \\
\hline Triplet chemotherapy & 1 & 0.7 & 4 & 3.0 \\
\hline \multicolumn{5}{|l|}{ Monoclonal Abs } \\
\hline Cetuximab use & 9 & 6.9 & 30 & 23.0 \\
\hline Panitumumab use & 1 & 0.7 & 4 & 3.0 \\
\hline Bevacizumab use & 21 & 16.1 & 46 & 35.3 \\
\hline
\end{tabular}

N: Number; LCC: left-sided colon carcinoma; RCC: right-sided colon carcinoma; NOS: not otherwise specified; DFS: disease-free survival; Monoclonal Abs: monoclonal antibodies.

log-rank test was used to assess differences between subgroups. Significance was defined at the $\mathrm{p}$ less than 0.05 level. The SPSS (version 21.0) was used for statistical analyses.

\section{Results}

The baseline patients' characteristics and treatment data regarding all eligible patients are summarized in Table I. Of the 130 patients considered eligible, $82(63.0 \%)$ were males and $48(36.9 \%)$ were female, with a median age of 63 years 
Right-sided

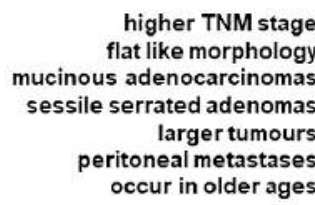

respond well to immunotherapy

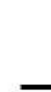 \\ is

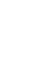

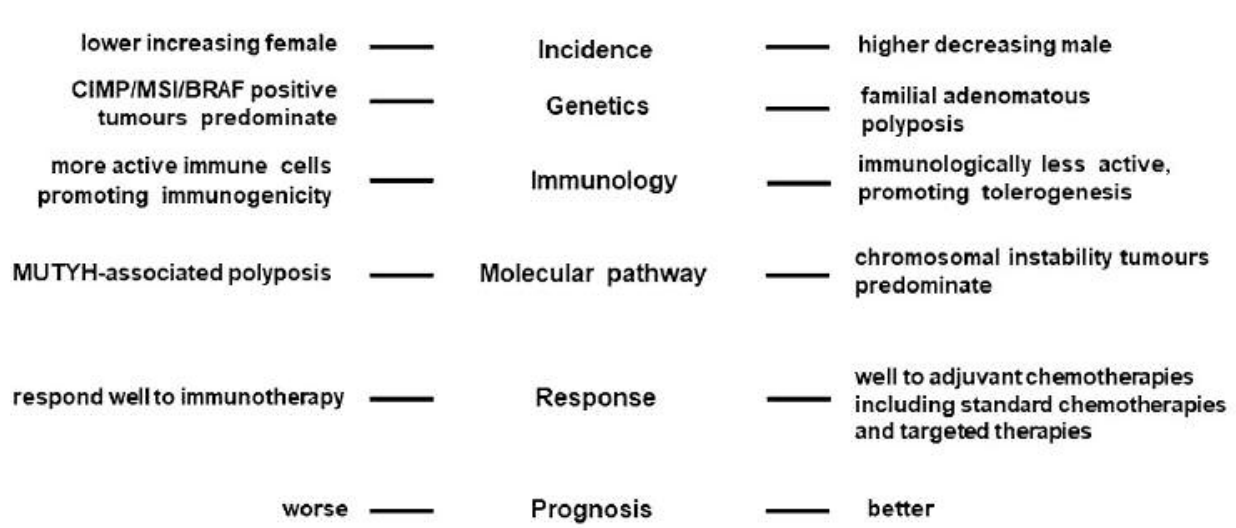

Left-sided

lower TNM stage

polypoid like morphology

tubular, villous

adenocarcinomas

smaller tumours

liver and lung metastases

occur in younger ages

Figure 1. Differences between right- and left-sided primary colon cancers.

(range $=40-82$ years). Regarding tumor location, $37(28.4 \%)$ patients had RCC, including $1(0.7 \%)$ appendix cancer, 10 (7.6\%) cecal cancers, 7 (5.3\%) ascending colon cancers, 3 $(2.3 \%)$ hepatic flexure colon cancers, $8(6.1 \%)$ transverse colon cancers and $9(6.9 \%)$ NOS (not otherwise specified) right colon cancers, whereas $93(71.5 \%)$ patients had LCC, including $1(0.7 \%)$ splenic flexure colon cancer, $9(6.9 \%)$ descending colon cancers, $22(16.9 \%)$ sigmoid colon cancers, $16(12.3 \%)$ rectum-sigmoid junction cancers, 29 $(22.3 \%)$ rectum cancers and $15(11.5 \%)$ NOS left colon cancers. As far as the mutational analysis of the RAS gene is concerned, 64 patients were wild-type (49.2\%) $(11.5 \%$ right-sided and $37.6 \%$ left-sided colon cancers) and 61 mutant $(46.9 \%)$ (16.1\% right-sided and $30.7 \%$ left-sided colon cancer) and 5 not tested (3.8\%). Metastatic disease sites included: liver only $23.8 \%$, liver + other $34.6 \%$ and the others $41.5 \%$. A total of 102 patients $(78.4 \%)$ had visceral metastases $v s$. 28 patients $(21.5 \%)$ without visceral metastases; 67 patients $(51.5 \%)$ had one metastatic site and $63(48.4 \%)$ had more than one metastatic site. The median duration of follow-up was 17.9 months (range=1-101 months). Nine patients $(6.9 \%)$ underwent neoadjuvant treatment and $47(36.1 \%)$ a post-operative adjuvant therapy. At the time of diagnosis, 72 patients $(55.3 \%)$ had already metastases, 10 patients $(7.6 \%)$ developed metastases within 6 months, 14 patients $(10.7 \%)$ between 6 and 12 months and 34 patients $(26.1 \%)$ after 12 months from surgery. A total of
$33.6 \%$ of patients were treated with anti-EGFR antibodies vs. $51.4 \%$ of patients treated with anti-VEGF antibody. In terms of responses to first-line treatment, disease progression was observed in 30 cases $(23.1 \%)$, partial responses in 43 $(33.1 \%)$ and stable diseases in $40(30.8 \%)$. The 2-yr OS for all pts was $80.7 \%$ (Figure 2A), $82.9 \%$ for LCC and $67.5 \%$ for RCC regardless of the treatment performed $(p=0.32)$ (Figure 2B). OS of LCC treated with anti-VEGF $(90.3 \%)$ was longer than LCC treated with anti-EGFR $(68.1 \%)$ $(p=0.18$ ) (Figure 2C) while in RCC treated was $74.2 \%$ with anti-VEGF and $64.3 \%$ with anti-EGFR $(p=0.25)$ (Figure 2D). The 1-yr mPFS for all patients was $40 \%$ (11 months, 95\% CI=9-12) (Figure 3A); it was longer in pts treated with anti-VEGF $v s$. anti-EGFR in LCC: $14(95 \% \mathrm{CI}=10-17)$ vs. 8 months (95\%CI=5-11) ( $p=0.06)$ (Figure 3B) and longer than just 2 months in RCC treated with anti-EGFR: 9 (95\%CI=117) $v s .7$ months $(95 \% \mathrm{CI}=3-12)(p=0.92)$ (Figure 3C). However, the 1-yr mPFS was statistically longer in LCC vs. RCC (46.8\% vs. 24.2\%, $p=0.0005)$ (Figure 3D), regardless of the therapy performed. The ORR (complete + partial responses) was $51.1 \%$ for left-sided and $25 \%$ in right-sided tumors $(p=0.008)$. We found a trend towards a higher ORR in RCC treated with anti-EGFR based therapy compared to anti-VEGF based therapy (50\% vs. $17.9 \%, p=0.09)$. The ORR was about the same in LCC treated with anti-EGFR vs. anti-VEGF based therapy ( $48.3 \%$ vs. $52.5 \%, p=0.71)$. A total of 11 complete responses (CRs) (12.5\%) were observed, all 

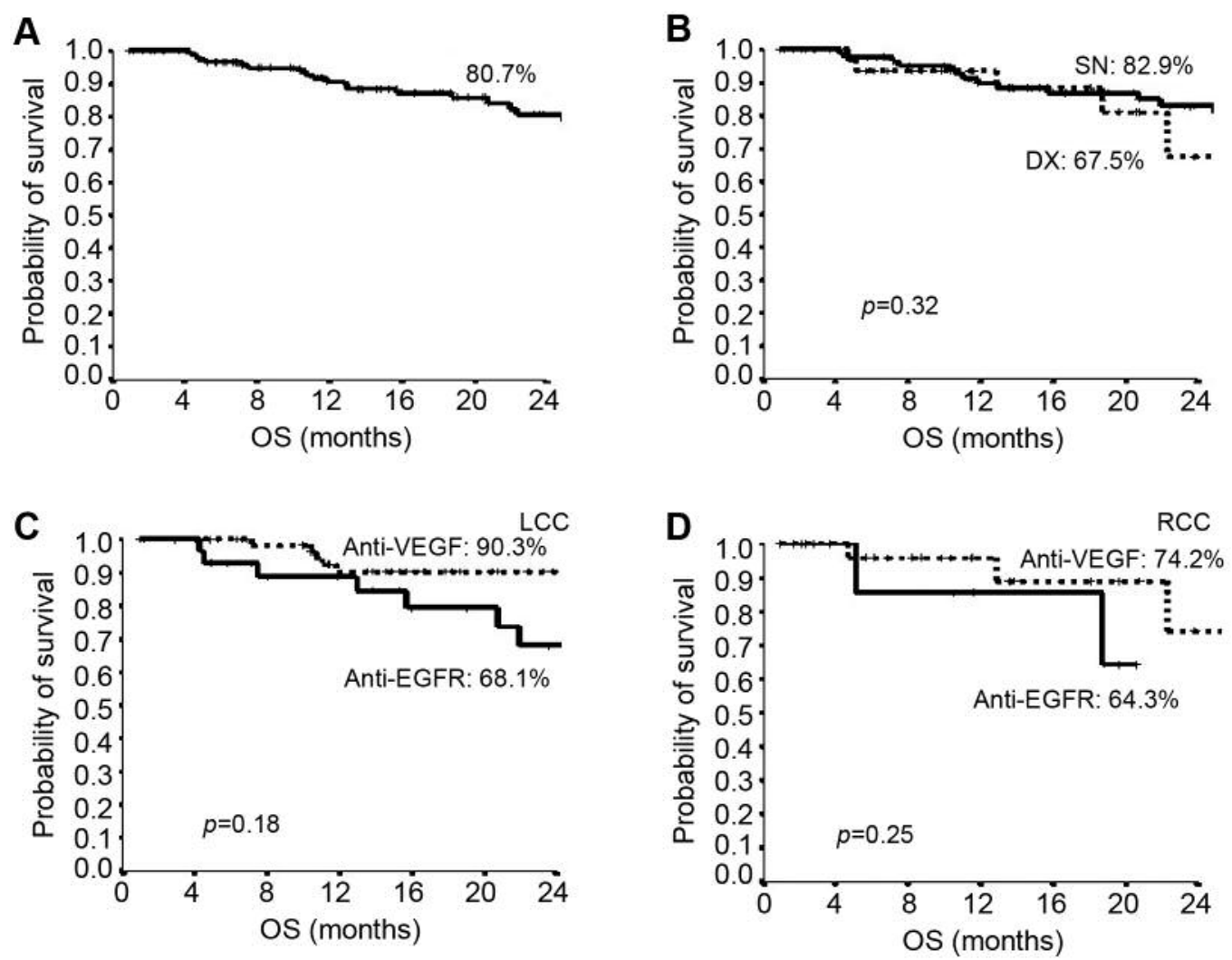

Figure 2. Overall survival (OS). (A) OS in all patients. (B) OS stratified by primary tumor site, regardless of the therapy performed. (C) OS stratified by primary tumor site (LCC) and therapy performed. (D) OS stratified by primary tumor site (RCC) and therapy performed.

in the left-sided ( $p=0.03): 8(13.6 \%)$ with anti-VEGF and 3 $(10.3 \%)$ with anti-EGFR. The $<6$ months and $>12$ months DFS post-adjuvant therapy were found better in RCC than LCC (respectively $33.3 \%$ vs. $13.3 \%$ and $66.7 \%$ vs. $57.8 \%$ ) whereas 6-12 months DFS was observed only in $28.9 \%$ of LCC, $p=0.056$ ). The efficacy outcomes are summarized in Table II.

\section{Discussion}

In our monocentric retrospective study, the possible prognostic and predictive impact of primary tumor location in patients with $\mathrm{mCRC}$ treated with first-line chemotherapy alone or in combination with anti-EGFR or anti-VEGF agents were assessed. So, we decided to examine, in our small clinical reality, the impact of the primary tumor location on prognosis and prediction of outcomes as well as its significance in guiding the choice of the most appropriate therapy for patients with mCRC in order to offer them the most beneficial, current, and novel therapies. To develop effective therapeutic regimens and improve therapeutic options, we also evaluated left and right-sided tumors as separate entities taking into account the differences between these tumors. First of all, regarding the relevance of the predictive value, the findings of the present analysis concur with previous reports and showed that treatment outcomes were better in patients with left-sided tumors than in those with right-sided tumors, indicating that the primary tumor location has a strong prognostic effect on survival irrespective of the chemotherapy and targeted agents used in first-line treatment. Our observations suggest that there is still a clinical need of novel treatment strategies for patients with right-sided mCRC. The literature data clearly indicate that RCC is associated with an inferior prognosis compared to LCC and that the prognostic value is evident both for first-line chemotherapy alone and chemotherapy plus targeted agents. Patients with RCC do not respond well to conventional chemotherapy, but show more promising results with immunotherapy because these cancers have high antigenic load. Longer survival is exhibited in LCC than RCC after palliative chemotherapies regardless of the treatment performed. Patients with LCC benefit more from adjuvant chemotherapy, such as 5-fluorouracil (5-FU) regimens, and from targeted therapies such as anti-EGFR therapy, and have a better prognosis. Our work confirms and emphasizes 

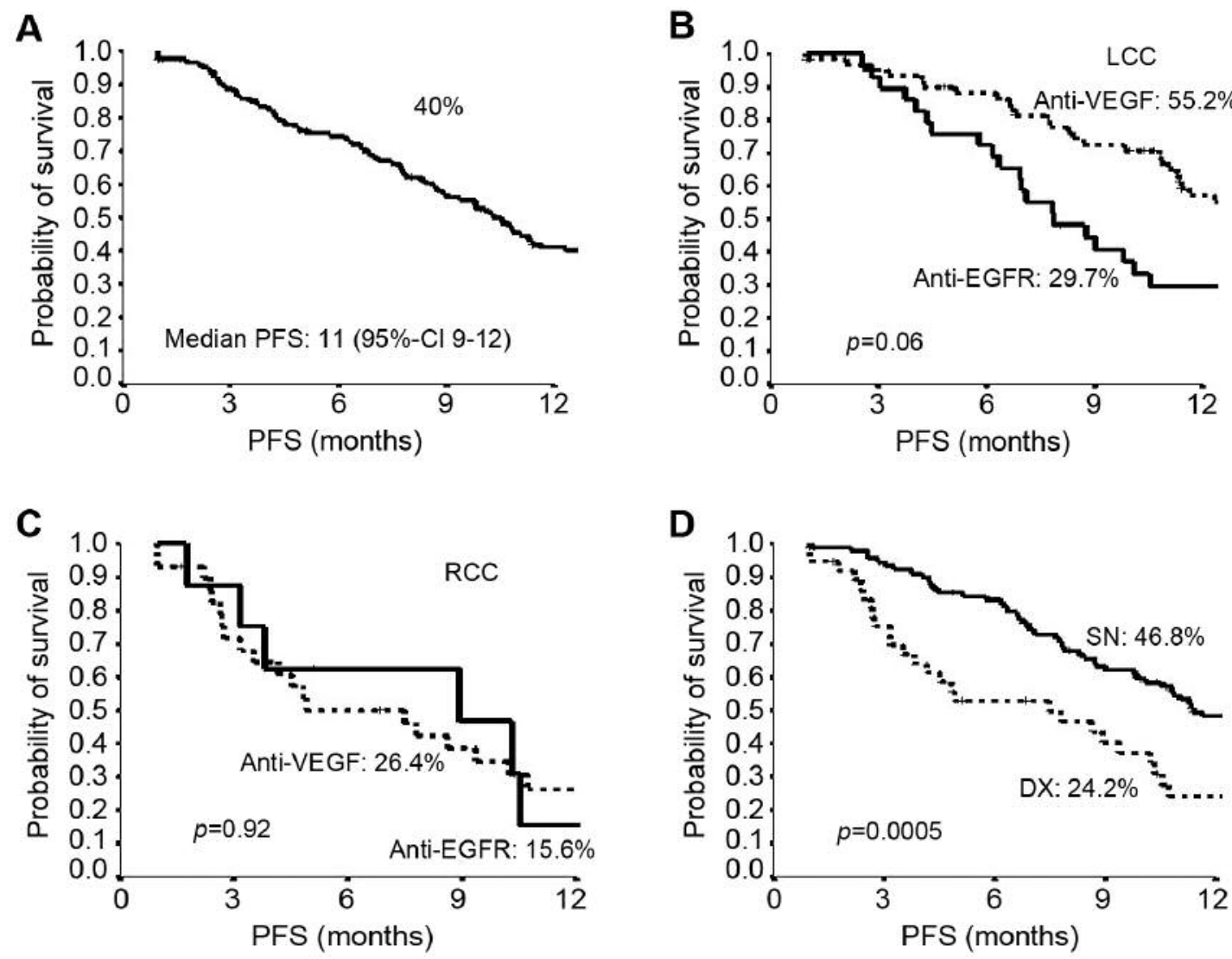

Figure 3. Progression-free survival (PFS). (A) PFS in all patients. (B) PFS stratified by primary tumor site (LCC) and therapy performed. (C) PFS stratified by primary tumor site $(R C C)$ and therapy performed. (D) PFS stratified by primary tumor site, regardless of the therapy performed.

Table II. Efficacy outcomes.

\begin{tabular}{|c|c|c|c|c|c|c|c|c|c|c|c|c|}
\hline & \multicolumn{2}{|c|}{ OS } & \multicolumn{4}{|c|}{ PFS } & \multicolumn{2}{|c|}{ ORR } & \multicolumn{4}{|c|}{ DFS post-adjuvant therapy } \\
\hline & $\begin{array}{l}2-\mathrm{yr} \\
(\%)\end{array}$ & $p$-Value & $\begin{array}{c}\text { mPFS } \\
\text { (months) }\end{array}$ & $95 \% \mathrm{CI}$ & $\begin{array}{l}1-\mathrm{yr} \\
(\%)\end{array}$ & $p$-Value & $\begin{array}{c}\mathrm{PR}+\mathrm{CR} \\
(\%)\end{array}$ & $p$-Value & $\begin{array}{c}<6 \\
\text { months }\end{array}$ & $\begin{array}{c}\text { 6-12 } \\
\text { months }\end{array}$ & $\begin{array}{c}>12 \\
\text { months }\end{array}$ & $p$-Value \\
\hline All patients & 80.7 & & 11 & $9-12$ & 40 & & 43.5 & 0.008 & 17.5 & 22.8 & 59.6 & 0.056 \\
\hline $\mathrm{RCC}$ & 67.5 & 0.32 & 7 & $2-13$ & 24.2 & 0.0005 & 25.0 & & 33.3 & - & 66.7 & \\
\hline LCC & 82.9 & & 11 & $9-13$ & 46.8 & & 51.1 & & 13.3 & 28.9 & 57.8 & \\
\hline \multicolumn{13}{|l|}{$\mathrm{RCC}$} \\
\hline Anti-EGFR Abs & 64.3 & 0.25 & 9 & $1-17$ & 15.6 & 0.92 & 17.9 & 0.09 & & & & \\
\hline Anti-VEGF Ab & 74.2 & & 7 & $3-12$ & 26.4 & & 50.0 & & & & & \\
\hline \multicolumn{13}{|l|}{ LCC } \\
\hline Anti-EGFR Abs & 68.1 & 0.18 & 8 & $5-11$ & 29.7 & 0.06 & 52.5 & 0.71 & & & & \\
\hline Anti-VEGF Ab & 90.3 & & 14 & $10-17$ & 55.2 & & 48.3 & & & & & \\
\hline
\end{tabular}

RCC: Right-sided colon cancer; LCC: left-sided colon cancer; CI: confidence interval; OS: overall survival; PFS: progression-free survival; RR: response rates; Anti-EGFR Abs: anti-EGFR antibodies; Anti-VEGF Abs: anti-VEGF antibody; DFS: disease-free survival; PD: progressive disease; PR: partial response; CR: complete response.

previous reports indicating an increasing importance of primary tumor location in daily clinical decision-making processes. We also confirmed that the provision of anti-VEGF alongside first-line chemotherapy is associated with improved
PFS outcomes in mCRC, as demonstrated in multiple clinical trials. Importantly for routine clinical practice, our small analysis with 130 patients with $\mathrm{mCRC}$ who received first line palliative chemotherapy with or without concurrent 
monoclonal antibodies, indicates an anti-VEGF efficacy regardless of primary tumor location. Based on current knowledge, reviewing the literature data, targeted therapies for mCRC have been developed and used in combination with conventional chemotherapy leading to improved overall survival. Some agents like bevacizumab, aflibercept, ramucirumab and regorafenib, specifically target the angiogenic pathways, others like cetuximab and panitumumab, target the EGFR pathway $(16,17)$. In the CALGB/SWOG 80405 study, patients who received cetuximab reached a median overall survival of 36 months for LCC and 16.7 months for RCC $(p<0.0001)$ (18). In addition, in the Canadian NCIC CO.17 study, LCC patients benefited more from cetuximab therapy than RCC patients (19). Similar results were obtained in the CRYSTAL and FIRE studies (20). In the PRIME study, panitumumab associated to FOLFOX regimen increased overall survival among LCC patients, but it was not effective in RCC patients (respectively, 32.5 months versus 23.6 months in LCC vs. 22.5 months versus 21.5 months in RCC) (21). Another commonly used targeted agent is bevacizumab that acts on the vascular endothelial growth factor (VEGFR) receptor. After treatment with bevacizumab, median overall survival was 31.4 months for LCC patients and 24.2 months for RCC patients. In the CALGB/SWOG 80405 study, patients with RCC benefited more from treatment with bevacizumab than from treatment with cetuximab (18). The association of bevacizumab and chemotherapy reduced mortality in both LCC and RCC patients, while the combination of cetuximab and chemotherapy was effective only in LCC patients (22). In addition, RCC patients with wild-type KRAS seem to benefit more from initial antiVEGF-based therapy and those with LCC benefit more from anti-EGFR treatment in association with chemotherapy (23, 24). Do not underestimate that the tumor location may also be an important variable that can optimize therapy strategies in the adjuvant setting (25). The primary tumor location is so important that influences the current NCCN and ESMO guidelines. Regarding first-line treatment of mCRC, NCCN guidelines recommend both EGFR and VEGF antibodies in LCC with wild-type RAS, whereas ESMO guidelines recommend EGFR antibodies in combination with chemotherapy in LCC with wild-type RAS. Both guidelines for RCC with mutant RAS, recommend chemotherapeutic combinations with VEGF antibodies (26, 27). Actually, available data do not support tumor location as a predictor of first-line anti-VEGF treatment efficacy. The tumor site is not an independent prognostic factor for OS and DFS rate after surgical treatment in colon carcinoma, whereas advanced $\mathrm{T}$ stage, node positivity, and poor histological grade are independent prognostic factors in oncologic outcomes. In summary, RCC tends to have more node positivity, poor histological grade and advanced $\mathrm{T}$ stage than the LCC. Thus, the prognosis of RCC is expected to be worse than LCC.
Summarizing and taking inspiration from our little experience, we conclude that colorectal cancer is not one disease but many. We also confirm that LCC seems to be a predictive factor for survival benefit from anti-EGFR therapy in patients with wild-type RAS tumors. On the other hand, RCC presents a poor response to the standard chemotherapy alone or in association with targeted agents. Anti-VEGF-based therapy is associated with a more favorable outcome in terms of PFS and OS. As in the literature, it was very interesting to find a trend towards a higher ORR in RCC treated with anti-EGFR therapy compared to anti-VEGF therapy; the data are, however, to be taken with caution due to the limited size of our sample and the retrospective nature of this study. Therefore, further investigations are surely needed.

\section{Conclusion}

Our observations underscore and confirm some of the previous studies on the sidedness of colorectal cancer patients; $\mathrm{mCRC}$ is a heterogeneous disease and primary tumor location has prognostic and predictive significance. Based on the results of our analysis, limited by heterogeneity of treatments and by the small sample size, we suggest a location-based therapy, with a benefit from the addition of anti-EGFR for wild-type $R A S$ left-sided tumors; complete responses and significantly better mPFS reached only in leftsided tumors regardless of the therapy performed. AntiVEGF therapy prolongs OS in patients with left-sided tumors and more aggressive treatments are needed for patients with right-sided tumors especially in advanced disease. Thus, we suppose that the primary tumor site of colon cancer should be considered in the clinical practice decision-making including treatment intensity in metastatic and locoregional settings. It should also represent an important stratification factor for future studies in the adjuvant therapy setting but should not impact on clinical decision making when considering the use of anti-VEGF. Anyway, larger studies and proper specialized treatment related to the location of colon cancer are needed to define if the type of chemotherapy backbone adopted and tumor location could be associated in order to improve treatment efficacy.

\section{Conflicts of Interest}

The Authors declare that they have no competing interests regarding this study.

\section{Authors' Contributions}

Conceptualization: CS MGC. Data Curation: CS. Formal Analysis: IS CS. Investigation: CS MGC SG PMA RMP TVR VVG MER FN EMR. Resources: CS MGC. Validation: CS. Visualization: CS. Writing - Original Draft Preparation: CS. Writing - Review \& Editing: EMR CS. All Authors have read and approved the final manuscript. 


\section{References}

1 Globocan 2018, Colorectal Cancer, Cancer Incidence and Mortality Worldwide: IARC Cancer Base No. 11, Lyon, France: International Agency for Research on Cancer. Available at http://globocan.iarc.fr (last accessed December 13, 2018)

2 Vatandoust S, Price TJ and Karapetis CS: Colorectal cancer: Metastases to a single organ. World J Gastroenterol 21(41): 11767-11776, 2015. PMID: 26557001. DOI: 10.3748/wjg.v 21.i41.11767

3 Benedix F, Kube R, Meyer F, Schmidt U, Gastinger I, Lippert H and Colon/Rectum Carcinomas Study Group: Comparison of 17,641 patients with right- and left-sided colon cancer: differences in epidemiology, perioperative course, histology, and survival. Dis Colon Rectum 53(1): 57-64, 2010. PMID: 20010352. DOI: 10.1007/DCR.0b013e3181c703a4

4 Petrelli F, Tomasello G, Borgonovo K, Ghidini M, Turati L, Dallera P, Passalacqua R, Sgroi G and Barni S: Prognostic survival associated with left-sided vs right-sided colon cancer: a systematic review and meta-analysis. JAMA Oncol 3(2): 211219, 2017. PMID: 27787550. DOI: 10.1001/jamaoncol. 2016.4227

5 Loupakis F, Yang D, Yau L, Feng S, Cremolini C, Zhang W, Maus MK, Antoniotti C, Langer C, Scherer SJ, Müller T, Hurwitz HI, Saltz L, Falcone A and Lenz HJ: Primary tumor location as a prognostic factor in metastatic colorectal cancer. J Natl Cancer Inst 107(3): dju427, 2015. PMID: 25713148. DOI: $10.1093 /$ jnci/dju427

6 Van Cutsem E, Nordlinger B, Adam R, Köhne CH, Pozzo C, Poston G, Ychou M, Rougier P and European Colorectal Metastases Treatment Group: Towards a pan-European consensus on the treatment of patients with colorectal liver metastasis. Eur J Cancer 42: 2212-2221, 2006. PMID: 169043 15. DOI: 10.1016/j.ejca.2006.04.012

7 Marzouk O and Schofield J: Review of histopathological and molecular prognostic features in colorectal cancer. Cancers (Basel) 3(2): 2767-2810, 2011. PMID: 24212832. DOI: 10.3390/ cancers 3022767

8 Nawa T, Kato J, Kawamoto H, Okada H, Yamamoto H, Kohno $\mathrm{H}$, Endo $\mathrm{H}$ and Shiratori Y: Differences between right- and leftsided colon cancer in patient characteristics, cancer morphology and histology. J Gastroenterol Hepatol 23(3): 418-423, 2008. PMID: 17532785. DOI: 10.1111/j.1440-1746.2007.04923.x

9 Carethers JM: One colon lumen but two organs. Gastroenterology 141(2): 411-412, 2011. PMID: 21708155. DOI: $10.1053 /$ j.gastro.2011.06.029

10 Bufill JA: Colorectal cancer: evidence for distinct genetic categories based on proximal or distal tumor location. AnnI Intern Med 113: 779-788, 1990. PMID: 2240880. DOI: 10.7326/0003-4819-113-10-779

11 Passardi A, Canale M, Valgiusti $\mathrm{M}$ and Ulivi P: Immune checkpoints as a target for colorectal cancer treatment. Int J Mol Sci 18(6): 1324, 2017. PMID: 28635639. DOI: 10.3390/ ijms 18061324

12 Mik M, Berut M, Dziki L, Trzcinski R and Dziki A: Right and left-sided colon cancer - clinical and pathological differences of the disease entity in one organ. Arch Med Sci 13(1): 157-162, 2017. PMID: 28144267. DOI: 10.5114/aoms.2016.58596

13 Baran B, Ozupek NM, Tetik NY, Acar E, Bekcioglu O and Baskin Y: Difference between left-sided and right-sided colorectal cancer: a focused review of literature. Gastroenterol Res 11(4): 264-273, 2018. PMID: 30116425. DOI: 10.14740/ gr1062w

14 Lee GH, Malietzis G, Askari A, Bernardo D, Al-Hassi HO and Clark SK: Is right-sided colon cancer different to left-sided colorectal cancer? - a systematic review. Eur J Surg Oncol 41: 300-308, 2015. PMID: 25468456. DOI: 10.1016/j.ejso. 2014.11.001

15 Yiu AJ and Yiu CY: Biomarkers in colorectal cancer. Anticancer Res 36(3): 1093-1102, 2016. PMID: 26977004.

16 Seeber A and Gastl G: Targeted therapy of colorectal cancer. Oncol Res Treat 39(12): 796-802, 2016. PMID: 27889786. DOI: $10.1159 / 000453027$

17 Jordan F, Grundmann N, Schenkirsch G, Märkl B, Messmann H, Anthuber M, Schmid C and Trepel M: Impact of primary tumor localization on the efficacy of bevacizumab in metastatic colorectal cancer. Anticancer Res 38(9): 5539-5546, 2018. PMID: 30194214. DOI: 10.21873/anticanres.12889

18 Venook AP, Niedzwiecki D, Innocenti F, Innocenti F, Fruth B, Greene C, O’Neil BH, Shaw JE, Atkins JN, Horvath LE, Polite BN, Meyerhardt JA, O'Reilly EM, Goldberg RM, Hochster HS, Blanke CD, Schilsky RL, Mayer RJ, Bertagnolli MM and Lenz HJ: Impact of primary tumor location on overall survival and progression-free survival in patients with metastatic colorectal cancer: analysis of CALGB/SWOG 80405 (Alliance). J Clin Oncol 34: 3504, 2016. DOI: 10.1200/JCO.2016. 34.15_suppl. 3504

19 Brule SY, Jonker DJ, Karapetis CS, O'Callaghan CJ, Moore MJ, Wong R, Tebbutt NC, Underhill C, Yip D, Zalcberg JR, Tu D and Goodwin RA: Location of colon cancer (right-sided versus left-sided) as a prognostic factor and a predictor of benefit from cetuximab in NCIC CO.17. Eur J Cancer 51(11): 1405-1414, 2015. PMID: 25979833. DOI: 10.1016/j.ejca.2015.03.015

20 Tejpar S, Stintzing S, Ciardiello F, Tabernero J, Van Cutsem E, Beier F, Esser R, Lenz HJ and Heinemann V: Prognostic and predictive relevance of primary tumor location in patients with RAS wild-type metastatic colorectal cancer: retrospective analyses of the CRYSTAL and FIRE-3 trials. JAMA Oncol 3(2): 194-201, 2017. PMID: 27722750. DOI: 10.1001/jamaoncol. 2016.3797

21 Boeckx N, Koukakis R, Op de Beeck K, Rolfo C, Van Camp G, Siena S, Tabernero J, Douillard JY, André T and Peeters M: Primary tumor sidedness has an impact on prognosis and treatment outcome in metastatic colorectal cancer: results from two randomized first-line panitumumab studies. Ann Oncol 28(8): 1862-1868, 2017. PMID: 28449055. DOI: 10.1093/ annonc/mdx 119

22 Aljehani MA, Morgan JW, Guthrie LA, Jabo B, Ramadan M, Bahjri K, Lum SS, Selleck M, Reeves ME, Garberoglio C and Senthil M: Association of primary tumor site with mortality in patients receiving bevacizumab and cetuximab for metastatic colorectal cancer. JAMA Surg 153(1): 60-67, 2018. PMID: 28975237. DOI: $10.1001 /$ jamasurg.2017.3466

23 Houts AC, Ogale S, Sommer N and Walker MS: Treatment patterns and outcomes in patients with KRAS wild type (WT) metastatic colorectal cancer $(\mathrm{mCRC})$ treated in first line with bevacizumab (B) or cetuximab (C) containing regimens. J Clin Oncol 34(4): 550, 2019. DOI: 10.1200/jco.2016.34.4_suppl.550

24 Lenz H, Lee F, Yau L, Koh H, Knost K, Mitchell EP, Bosanac I, Mancao $\mathrm{C}$ and Parikh A: MAVERICC, a phase 2 study of 
mFOLFOX6 bevacizumab (BV) vs FOLFIRIBV with biomarker stratification as firstline (1L) chemotherapy (CT) in patients (pts) with metastatic colorectal cancer (mCRC). J Clin Oncol 34(Suppl 4S): abstr 493, 2016. DOI: 10.1200/jco.2016. 34.4_suppl.493

25 Peng J, Li C, Wang F, Zhang H, Xiao W, Li H, Lu Z, Pan Z, Wu $\mathrm{X}$ and Zhang R: Right- and left-sided stage III colon cancers present different prognostic outcomes of oxaliplatin-based adjuvant chemotherapy after curative resection. Cancer Manag Res 10: 2095-2103, 2018. PMID: 30140160. DOI: 10.2147/ CMAR.S163520

26 NCCN Clinical Practice Guidelines in Oncology. Colon Cancer Version 2. 2019 - May 15, 2019.

27 Van Cutsem E, Cervantes A, Adam R, Sobrero A, Van Krieken JH, Aderka D, Aranda Aguilar E, Bardelli A, Benson A, Bodoky G, Ciardiello F, D'Hoore A, Diaz-Rubio E, Douillard JY, Ducreux M, Falcone A, Grothey A, Gruenberger T, Haustermans
K, Heinemann V, Hoff P, Köhne CH, Labianca R, Laurent-Puig P, Ma B, Maughan T, Muro K, Normanno N, Österlund P, Oyen WJ, Papamichael D, Pentheroudakis G, Pfeiffer P, Price TJ, Punt C, Ricke J, Roth A, Salazar R, Scheithauer W, Schmoll HJ, Tabernero J, Taïeb J, Tejpar S, Wasan H, Yoshino T, Zaanan A and Arnold D: ESMO consensus guidelines for the management of patients with metastatic colorectal cancer. Ann Oncol 27: 1386-1422, 2016. PMID: 27380959. DOI: 10.1093/annonc/ mdw235

Received July 27, 2019

Revised August 4, 2019

Accepted August 5, 2019 